\title{
CALIDAD DEL CUIDADO DE ENFERMERÍA DESDE EL ENFOQUE DE DONABEDIAN EN PACIENTES HOSPITALIZADOS CON DOLOR
}

\author{
QUALITY OF NURSING CARE FROM THE DONABEDIAN APPROACH \\ IN HOSPITALIZED PATIENTS WITH PAIN
}

\section{QUALIDADE DO CUIDADO DE ENFERMAGEM A PARTIR DA ABORDAGEM DE DONABEDIAN EM PACIENTES HOSPITALIZADOS COM DOR}

\author{
Alma Delia Santiago Mijangos* \\ Esther Alice Jiménez Zúñiga** \\ Martha Pérez Fonseca*** \\ Amisaday Hernández Martínez****
}

\begin{abstract}
RESUMEN
Objetivo: Evaluar la calidad del cuidado de enfermería, desde el enfoque de Donabedian, en pacientes hospitalizados con dolor. Material y Método: Estudio descriptivo transversal realizado en un hospital de segundo nivel del municipio de Minatitlán, Veracruz, México, entre octubre y noviembre de 2018. Se empleó muestreo no probabilístico por conveniencia con una muestra de 73 enfermeros/as que, previo consentimiento informado, respondieron el instrumento "Estándares e indicadores que miden el procedimiento en el control del dolor-valoración”, compuesto por datos sociodemográficos, laborales y 24 ítems que miden la evaluación de la estructura, proceso y resultado de la atención, desde el enfoque de Donabedian. Se utilizó análisis descriptivo e inferencial como Chi-cuadrado, $U$ de Mann-Whitney y Kruskal-Wallis. Resultados: La media de edad fue 41,22 ( \pm 10,027 DS) y el 41,1\% mostró más de 12 años de antigüedad laboral. Se obtuvo calidad máxima en la evaluación de estructura $(=90,14 ; \pm 8,68 \%)$, proceso $(=92,97 ; \pm 9,55 \%)$ y resultados $(=93,05 ; \pm 9,50 \%)$; no hubo asociación entre la variable calidad del cuidado de enfermería con la edad, sexo, turno, tipo de servicio, antigüedad laboral y tiempo de laborar en el servicio ( $\mathrm{p}>0,05)$, pero sí hubo asociación con el nivel académico $(\mathrm{p}<0,05)$. Además, se encontró que existen diferencias estadísticamente significativas entre la calidad del cuidado de enfermería de acuerdo con el nivel académico y la edad $(\mathrm{p}<0,05)$. Conclusiones: El personal de enfermería requiere de un desarrollo profesional continuo para mejorar la calidad del cuidado de enfermería
\end{abstract}

\footnotetext{
*Enfermera. Doctora en Educación, Facultad de Enfermería de la Universidad Veracruzana, Minatitlán, Veracruz, México. orcid. org/0000-0003-3596-5526 Email: alsantiago@uv.mx Autor de correspondencia.

**Enfermera. Doctora en Ciencias de Enfermería, Facultad de Enfermería de la Universidad Veracruzana, Minatitlán, Veracruz, México. orcid.org/0000-0002-8264-0903 Email: esjimenez@uv.mx

***Enfermera. Maestra en Ciencia de Enfermería, Facultad de Enfermería de la Universidad Veracruzana, Minatitlán, Veracruz, México. orcid.org/0000-0002-8709-0875 Email: marperez@uv.mx

****Enfermera. Licenciada en Enfermería, Facultad de Enfermería de la Universidad Veracruzana, Minatitlán, Veracruz, México. orcid.org/0000-0001-7487-1632 Email: amisaday.Hdz@hotmail.com
} 
en pacientes hospitalizados con dolor, que se refleje en la evaluación de la estructura, proceso y resultado de la atención de las instituciones de salud.

Palabras clave: Calidad; Cuidado de Enfermería; Pacientes; Dolor.

\begin{abstract}
Objective: To evaluate the quality of nursing care, from the Donabedian approach, in hospitalized patients with pain. Materials and Methods: Descriptive cross-sectional study carried out in a second level hospital in the municipality of Minatitlán, State of Veracruz, Mexico, between October and November 2018. Nonprobabilistic convenience sampling with a sample consisting of 73 female and male nurses, who after prior informed consent, answered the instrument "Estándares e indicadores que miden el procedimiento en el control del dolor-valoración", made up of sociodemographic and work-related data, as well as 24 items that measure the evaluation of the structure, process and outcome of care, from the Donabedian approach. Descriptive and inferential analysis such as Chi-square, Mann-Whitney U and Kruskal-Wallis, was used. Results: The average age of the participants was $41.22( \pm 10,027 \mathrm{SD})$ and $41.1 \%$ showed more than 12 years of length of service. Maximum quality was obtained in the evaluation of the structure (=90.14 $\pm 8.68 \%)$, process $(=92.97 \pm 9.55 \%)$, and results $(=93.05 \pm 9.50 \%)$; there was no association between the variable quality of nursing care and age, sex, shift, type of service, length of service, and time in service ( $p>0.05)$, however, there was an association with academic level $(\mathrm{p}<0.05)$. Additionally, it was found that there are statistically significant differences between the quality of nursing care according to academic level and age $(p<0.05)$. Conclusions: Nursing staff require continuous professional development to improve the quality of nursing care in hospitalized patients with pain that is reflected in the structure, process and outcome of care in health institutions.
\end{abstract}

Key words: Quality; Nursing Care; Patients; Pain.

\title{
RESUMO
}

Objetivo: Avaliar a qualidade do cuidado de enfermagem, a partir da abordagem de Donabedian, em pacientes hospitalizados com dor. Material e método: Estudo descritivo transversal realizado em um hospital de segundo nível do Município de Minatitlán, Veracruz, México, entre outubro e novembro de 2018. Foi utilizada uma amostragem não probabilística por conveniência com uma amostra de 73 enfermeiros/as que, com consentimento informado prévio, responderam o questionário "Estándares e indicadores que miden el procedimiento en el control del dolor-valoración", composto por dados sociodemográficos, trabalhistas e 24 itens que medem a avaliação da estrutura, processo e resultado da atenção, a partir da abordagem de Donabedian. Utilizou-se análise descritiva e inferencial como Chi-quadrado, U de Mann-Whitney e KruskalWallis. Resultados: A média de idade foi de 41,22 ( \pm 10,027 DS) e um 41,1\% apresentaram mais de 12 anos de antiguidade no trabalho. A máxima qualidade foi obtida na avaliação da estrutura $(=90,14 ; \pm 8,68 \%)$, processo (= 92,97; $\pm 9,55 \%)$ e resultados (=93,05; $\pm 9,50 \%)$; não houve associação entre a variável qualidade do cuidado de enfermagem com a idade, sexo, turno, tipo de serviço, antiguidade no trabalho e tempo de atuação no serviço $(p>0,05)$, mas houve associação com o nível acadêmico $(p<0,05)$. Além disso, descobriu-se que existem diferenças estatisticamente significativas entre a qualidade do cuidado de enfermagem de acordo com o nível acadêmico e a idade $(\mathrm{p}<0.05)$. Conclusóes: A equipe de enfermagem requer um desenvolvimento profissional contínuo para melhorar a qualidade do cuidado de enfermagem em pacientes hospitalizados com dor, que se reflete na avaliação da estrutura, processo e resultado da atenção das instituiçóes de saúde.

Palavras-chave: Qualidade; Cuidados de Enfermagem; Pacientes; Dor.

Fecha de recepción: 6/08/2020

Fecha de aceptación: 14/10/2020 


\section{INTRODUCCIÓN}

La calidad para la Organización Mundial de la Salud (OMS) es realizar una actividad con estándares de intervenciones de probada seguridad, de fácil acceso a la población y que a su vez tiene la capacidad de producir un impacto en la morbilidad, discapacidad, mal nutrición y muerte de las personas, logrando el mejor resultado con el mínimo de daños y la máxima satisfacción del paciente. Es así como el término calidad, aplicado al área de salud, significa comparar, evaluar y establecer mejoras en la prestación de servicios en salud $^{(1,2)}$.

En los servicios de salud la calidad se refleja en la pertinencia del conocimiento actual del profesional de enfermería y de los resultados favorables en la salud de las personas. Diversos autores han realizado aportaciones a este tema, entre ellos destaca Donabedian, quien define a la calidad de la atención como "el modelo de asistencia esperado para maximizar el nivel de bienestar del paciente, una vez tomado en cuenta el balance de beneficios y pérdidas esperados en todas las fases del proceso asistencial"(3), lo que hace referencia a que la calidad, tanto en el campo de la salud como en otras áreas del conocimiento, se aplica a la excelencia de una disciplina, a la perfección de un proceso, a la obtención de buenos resultados de una técnica o de un procedimiento, en otras palabras, "hacer bien las cosas correctas"(2).

Lo antes mencionado guarda estrecha relación con el concepto de cuidado, término que a lo largo del tiempo se ha proclamado como la esencia de enfermería y que se expresa de forma explícita e implícita en el ejercicio profesional. Teóricas de enfermería destacan que el cuidado ha trascendido según el enfoque de estudio y con un impacto recíproco hacia quien da el cuidado y en quién lo recibe, en por qué y para qué del cuidado, en dónde y cuándo se da ese cuidado, y en el cómo y con qué se cuida. Además, en ese momento de cuidado se reconoce la realidad del otro y existe una relación de intercambio en donde el cuidado no significa curar, sino que es el medio para lograr el bienestar de la persona ${ }^{(4)}$.

Así, por ejemplo, la teoría general de Orem es un modelo que enfatiza que los cuidados de enfermería representan un servicio especializado centrado en las personas que sufren incapacidades para autocuidarse, entonces el papel de enfermería es apoyar a la persona para que avance en la mejora de su estado de salud hasta que sea capaz de su propio cuidado. En este proceso la enfermera hace uso de tres formas de actuación propuestos por Orem: Sistema de intervención totalmente compensatorio, parcialmente compensatorio y de apoyo educativo, según sea la condición del paciente, así como de su voluntad para recibir el cuidado, como es el caso de los pacientes con dolor ${ }^{(5)}$.

Es así como la enfermería se ha destacado por organizar, planificar, priorizar y mostrar dominio de las intervenciones dirigidas al cuidado de los pacientes, con una base de conocimientos científicos y también de la persona, sin embargo, en la actualidad el cuidado en las instituciones de salud se encuentra inmerso en una atmósfera de tensión y conflicto entre un deber ser del cuidado orientado hacia la empatía con el otro y, por otra parte, un sistema que exige eficacia y eficiencia sin contar con los recursos necesarios. Cuando esto pasa, el cuidado se centra en la rutina de procedimientos en un tiempo determinado, dejando de lado lo esencial del cuidado que es la persona ${ }^{(6-8)}$.

A pesar de esto, la labor de enfermería es hacer el bien al paciente al brindar un cuidado de calidad que permita satisfacer sus necesidades en salud, en este caso, para aliviar y reducir el dolor de las personas durante su estancia en el hospital.

Diversos autores ${ }^{(9,10)}$ han realizado trabajos relacionados con la calidad del cuidado de enfermería, desde la percepción del paciente, empleando el Cuestionario de calidad de cuidados de enfermería (Cucace), versión en castellano de Alonso et al. ${ }^{(11)}$ o versión en inglés de Thomas et al. ${ }^{(12)}$ y otros ${ }^{(4,13)}$, han aplicado el Care-Q de Patricia Larson ${ }^{(14)}$.

Estas investigaciones destacan que la calidad tiene diferentes concepciones dependiendo del contexto y/o el área de salud y que está conformada por aspectos fundamentales, como lo técnico, la ética, lo interpersonal y de percepción.

La técnica supone hacer uso de la tecnología de la forma más adecuada y que contribuya el restablecimiento de la salud del enfermo ${ }^{(15)}$. La ética basada en las normas, principios y valores, rigen el ejercicio del profesional de enfermería, además de tener como eje rector a la bioética, que permite tomar decisiones trascendentales para la vida del paciente y de sus cuidados, y así aliviar el dolor y evitar el sufrimiento teniendo en cuenta 
los principios de autonomía, beneficencia, no maleficencia y de justicia ${ }^{(16,17)}$. La interpersonal es establecer un vínculo de respeto, que satisface tanto al profesional como al paciente, por lo tanto, el personal de enfermería se centra en la persona más que en la enfermedad. La parte perceptual es la experiencia dicha por el paciente, que en su mayoría nada tiene que ver con los aspectos procedimentales, sino con el trato humano ${ }^{(15)}$.

Estos cuatro aspectos tienen la misma importancia y son indispensables para lograr una atención eficiente, eficaz y efectiva, que a su vez son evaluados bajo tres enfoques propuestos por Donabedian: Primero, la evaluación de la estructura, que consiste en la evaluación de los instrumentos de atención y de su organización, que incluye la infraestructura de los servicios, recursos humanos y materiales, es el principal elemento tomado en cuenta por los organismos de certificación y acreditación y cuando se cumplen ciertas condiciones específicas es probable que marchen por buen camino ${ }^{(18)}$; segundo, la evaluación del proceso que consiste en evaluar el cuidado en sí, donde se someten a juicio profesional los elementos y detalles de la atención desde los estándares hasta los procesos y políticas, acordes a normas oficiales; por último la evaluación de los resultados, generalmente especificada en términos de salud del paciente, bienestar y satisfacción. La medida en que se logran los resultados deseados acordados, es la prueba definitiva de los supuestos inherentes al uso de la estructura y del proceso en la evaluación de la atención ${ }^{(18)}$.

Si bien en México se trabaja en prestar servicios de salud de calidad, que se ven reflejados a partir del siglo XX, y la enfermera brinda cuidados para disminuir o aliviar el dolor en los hospitales, aún no se considera el dolor como un procedimiento que se tiene que tomar en cuenta para la mejora de la calidad institucional de los cuidados de enfermería $^{(19)}$.

El dolor que, según la International Association for the Study of Pain (IASP), es una experiencia sensorial y emocional desagradable asociada con daño tisular real o potencial, o descrita en términos de dicho daño ${ }^{(20)}$, requiere cuidados especiales, donde enfermería juega un papel relevante por ser quien identifica los factores que aumentan o disminuyen el dolor y planifica las intervenciones necesarias para disminuirlo. El cuidado de enfermería debe reconocer la presencia de la experiencia dolorosa en el paciente hospitalizado y no se debe pasar por alto en los hospitales locales.

En este contexto, en México se carece de investigaciones respecto al servicio proporcionado a los usuarios hospitalizados con dolor, por lo que la pregunta de investigación que orientó la presente investigación fue: ¿Cuál es la calidad del cuidado de enfermería, desde el enfoque de Donabedian, en pacientes hospitalizados con dolor y con qué variables sociodemográficas y laborales está asociada esa calidad? Para responder a lo anterior, primero se caracterizó a las enfermeras profesionales respecto a variables sociodemográficas y laborales, y se conoció la calidad del cuidado según las dimensiones de estructura, proceso y resultado propuestos por Donabedian.

\section{MATERIAL Y MÉTODO}

Tipo de estudio y unidad de análisis: Se trata de un estudio de tipo cuantitativo, no experimental, transversal analítico que se realizó entre el 11 de octubre y el 2 de noviembre de 2018, en profesionales de enfermería en turnos matutino y vespertino, de un hospital público de segundo nivel del municipio de Minatitlán, Veracruz, México.

Muestra: Para el cálculo de la muestra se tomó en cuenta a una población de 89 profesionales de enfermería, con base en un muestreo no probabilístico por conveniencia (debido a que no se contó con una lista de la población a estudiar) y evitar los posibles sesgos con el tamaño de la muestra, se realizó el cálculo muestral para poblaciones finitas con un mínimo de enfermeros que participarían en el estudio, considerando un nivel de confianza de 95\% y error muestral de 5\%. Los criterios de inclusión fueron: ser enfermero/a de cada uno de los servicios del hospital con más de seis meses de antigüedad, de ambos sexos, sin distinción del nivel académico y estar en condiciones físicas favorables para responder al instrumento. Se excluyó al personal de enfermería que no contestó el instrumento en su totalidad, que fuera pasante de enfermería o contara con un permiso temporal al momento de aplicar el instrumento. La muestra final quedó conformada por 73 enfermeros. 
Recolección de datos: Para la aplicación del instrumento, los profesionales fueron abordados en el hospital y se les invitó a participar explicándoles el objetivo de la investigación. Si ellos aceptaban se les solicitaba el consentimiento informado en donde declararon colaborar en la investigación en forma voluntaria. También se les garantizó el anonimato en sus respuestas, explicándoles previamente que los datos obtenidos serían utilizados para la descripción de la calidad del cuidado de enfermería brindado por profesionales y por tanto la información sería de carácter confidencial y privada.

El instrumento fue autoadministrado e incluyó variables sociodemográficas (edad y sexo) y laborales (turno y servicio asignado). La variable calidad del cuidado se midió a través de la escala "Estándares e indicadores que miden el procedimiento en el control del dolor-valoración"(21), conformada por un total de 24 ítems, divididos en tres dimensiones que indagan respecto a estándares de estructura, proceso y resultado. La estructura incluyó indicadores como: nivel académico de la enfermera, antigüedad laboral y tiempo en el servicio, uso del diagnóstico de enfermería, diagnóstico médico, tipo de usuario, material y equipo.

En el análisis del proceso se especifican los pasos que se aplican en el procedimiento para controlar el dolor por parte de enfermería, con indicadores como: valoración al usuario, identificación del principio científico, tiempo para escuchar las quejas de los clientes sobre algún tipo de dolor, alienta a los clientes para que reporten la presencia del dolor, investiga inmediatamente sobre el reporte de dolor que le hizo el cliente, emplea escalas de valoración del dolor sobre todo en recién nacidos o pediátricos; localiza e identifica el tipo de dolor que presenta el usuario. De igual forma si presta atención a los comportamientos que comúnmente se asocian al dolor, ejemplo: llanto, expresión facial de tensión, ansiedad, posición o movimiento poco usual, diaforesis, cambios en los signos vitales, etc.; expone al médico los datos obtenidos sobre el dolor; emplea técnicas como el confort de la cama, meditación, masaje, etc. para aliviar el dolor; ministra analgésicos de acuerdo a indicaciones médicas; nuevamente evalúa el dolor para identificar si este ha disminuido, y reporta en la hoja de enfermería datos como: tipo de dolor, la intensidad y tratamiento administrado al cliente.
En el estándar de resultados se integraron indicadores como: realización de procedimiento acorde a los principios científicos, información al usuario de los procedimientos a realizar, seguridad del personal de enfermería al hacer los procedimientos y si evalúa al concluir.

Cada pregunta, en una escala tipo Likert, está integrada por tres respuestas, donde 1 es igual a siempre, 2 a algunas veces y 3 a nunca. Se considera que el/la enfermero/a alcanza una calidad máxima del cuidado si realiza el procedimiento con el 90 a $100 \%$ de los indicadores con índice de capacidad máxima; tiene calidad regular del cuidado si el/ la enfermero/a realiza el procedimiento con el 80 a $89 \%$ de los indicadores con índice de capacidad máxima y tiene calidad mínima del cuidado si realiza el procedimiento con menos o igual al 79\% de los indicadores con índice de capacidad máxima, e indica que el/la enfermero/a tiene que reforzar los procedimientos teórico-prácticos. $\mathrm{El}$ instrumento tuvo, en este estudio, un alfa de Cronbach de 0,76.

Análisis de datos: Los datos obtenidos fueron procesados a través del programa estadístico Statistic Package for the Social Science (SPSS) versión 24.0 para Windows, del cual se obtuvo estadística descriptiva como: frecuencia, porcentaje y medidas de tendencia central como la media y desviación estándar. Además, se empleó estadística no paramétrica con la prueba de Chi cuadrada para conocer la existencia de asociación entre variables nominales y ordinales; la prueba U Mann-Whitney para contrastar el sexo y el turno, y la prueba Kruskal-Wallis para comparar más de tres grupos independientes. En caso de que la hipótesis nula resultara en rechazó se aplicó la prueba a posteriori. Se tomó en cuenta una significancia estadística con un valor de $\mathrm{p} \leq 0,05$.

Esta investigación se realizó tomando en cuenta la Declaración Mundial de Helsinki ${ }^{(22)}$ y el Reglamento de la Ley General en Materia de Investigación para la Salud de México ${ }^{(23)}$, por lo que el presente estudio se catalogó como investigación sin riesgo, ya que no se realizó ninguna intervención o modificación intencionada en las variables fisiológicas, psicológicas y sociales de las personas. Además, fue respaldada con informe del Comité de Ética de la Facultad de Enfermería de la Universidad Veracruzana, registro CIEE-ECS 167. 


\section{RESULTADOS}

Características sociodemográficas y laborales: La edad promedio del personal de enfermería fue de 41,22 ( $\pm 10,027)$ años con un rango mínimo de 22 y máximo de 57. En la Tabla 1 se observa que la mayoría estaba en el rango etario de 40 a 49 años $(30,1 \%)$, eran mujeres $(79,5 \%)$ en turno matutino (57,5\%), trabajaba en el servicio de Urgencias $(27,4 \%)$, con más de 12 años de antigüedad laboral $(41,1 \%)$ y más de 7 años en el servicio $(35,6 \%)$.

Calidad del cuidado de enfermería en pacientes con dolor: La media de la calidad fue de $92,37 \%$ $( \pm 8,09 \%)$ con un rango mínimo de 69 y máximo de 100. En cuanto a la calidad del cuidado de enfermería, de acuerdo a los tres enfoques propuestos por Donabedian, se obtuvo que la evaluación de estructura tuvo una media de 90,14 ( $\pm 8,68 \%)$ con una mínima de 66 y máxima de 100; la evaluación de proceso con una media de $92,97( \pm 9,55 \%)$ con una mínima de 58 y máxima de 100 y la evaluación de resultados con una media de 93,05 ( \pm 9,50\%) con una mínima de 58 y máxima de 100.

La calidad del cuidado de enfermería en pacientes con dolor en la dimensión de resultados fue la más valorada y la de estructura la menos valorada de acuerdo con la media, sin embargo, las tres dimensiones tuvieron una calidad máxima del cuidado. Además, de acuerdo con la Tabla 2, el 74\% presentó calidad máxima, aunque hubo un 11\% con calidad mínima en la dimensión estructura; en cuanto a la dimensión de proceso, el $76,7 \%$ tuvo calidad máxima y un $13,7 \%$ calidad mínima; en la dimensión resultados el 78,1\% obtuvo calidad máxima. Así la dimensión mejor evaluada fue la de resultados y con más calidad mínima fue la de proceso.

Asociación entre calidad del cuidado de enfermería en pacientes con dolor y variables sociodemográficas y laborales: En la Tabla 3 se observa que hubo calidad máxima en el personal de 50 y más años $(85,7 \%)$, en las mujeres $(75,9 \%)$, en el turno vespertino $(77,4 \%)$ y en el servicio de cirugía (85,7\%). Además, se puede observar que en la variable nivel académico hay un nivel de significancia menor que $0,05\left(\mathrm{X}^{2}=0,000<0,05\right)$, lo que indica que existe una asociación entre la calidad del cuidado de enfermería en pacientes con dolor y el nivel académico del personal de enfermería.

Así también en la Tabla 4 al analizar con la prueba U Mann-Whitney las variables sexo y turno con la calidad del cuidado de enfermería, se observó que no existen diferencias estadísticamente significativas. Al contrastar con la prueba KruskalWallis las variables: edad, nivel académico, servicio, antigüedad y años en el servicio, se observó que existen diferencias, estadísticamente significativas, entre la calidad del cuidado de enfermería y las variables edad $(\mathrm{p}=0,027<0,05)$ y nivel académico $(\mathrm{p}=0,001<0,05)$, es decir, la calidad del cuidado de enfermería depende de la edad y el nivel académico.

Al existir diferencias significativas en las variables edad y nivel académico se aplicó la prueba Kruskal-Wallis a posteriori como lo muestra la Tabla 5, en donde la calidad del cuidado de un/a enfermero/a general $(p=0,015<0,05)$ con rango medio de 44,59, un/a licenciado/a en enfermería $(\mathrm{p}=0,003<0,05)$ con un rango medio de 41,42 o un/a enfermero/a con especialidad de posgrado $(\mathrm{p}=0,034<0,05)$ con un rango medio de 42 no es la misma que la otorgada por un auxiliar de enfermería (rango medio de 17,74) a un paciente con dolor, existiendo diferencias significativas a favor del/la enfermero/a general, porque el rango medio es superior.

Se observó también que la calidad del cuidado de enfermería de un/a enfermero/a de 50 años y más $(\mathrm{p}=0,029<0,05)$ con rango medio de 48,52 no es la misma que la otorgada por los/as enfermeros/as de 40-49 ańos con rango medio de 30,39 ni con los de 22-29 años con rango medio de 33,32, ni con los de 30-39 ańos con rango medio de 34,05 a un paciente con dolor, existiendo diferencias significativas a favor de los/las enfermeros/as con edad de 50 años y más. 
Tabla 1. Características sociodemográficas y laborales de los/as enfermeros/as participantes ( $\mathrm{n}=73$ ).

\begin{tabular}{|c|c|c|c|}
\hline Variable & Categorías & Fr & $\%$ \\
\hline \multirow[t]{4}{*}{ Edad (años) } & $22-29$ & 11 & 15,1 \\
\hline & $30-39$ & 19 & 26,0 \\
\hline & $40-49$ & 22 & 30,1 \\
\hline & 50 y más & 21 & 28,8 \\
\hline \multirow[t]{2}{*}{ Sexo } & Mujer & 58 & 79,5 \\
\hline & Hombre & 15 & 20,5 \\
\hline \multirow[t]{2}{*}{ Turno } & Matutino & 42 & 57,5 \\
\hline & Vespertino & 31 & 42,5 \\
\hline \multirow[t]{6}{*}{ Servicio } & Cirugía & 7 & 9,6 \\
\hline & Medicina interna & 14 & 19,2 \\
\hline & Recuperación & 12 & 16,4 \\
\hline & Urgencias & 20 & 27,4 \\
\hline & Traumatología & 3 & 4,1 \\
\hline & Ginecología & 17 & 23,3 \\
\hline \multirow[t]{6}{*}{ Nivel académico } & Auxiliar de enfermería & 17 & 23,3 \\
\hline & Enfermera general & 11 & 15,1 \\
\hline & Especialidad postécnico & 1 & 1,4 \\
\hline & Especialidad de posgrado & 12 & 16,4 \\
\hline & Licenciatura & 31 & 42,5 \\
\hline & Maestría & 1 & 1,4 \\
\hline \multirow[t]{5}{*}{ Antigüedad laboral } & Menos de 3 años & 8 & 11,0 \\
\hline & 4-6 años & 12 & 16,4 \\
\hline & 7-9 años & 12 & 16,4 \\
\hline & 10-12 años & 11 & 15,1 \\
\hline & 12 ó más años & 30 & 41,1 \\
\hline \multirow[t]{5}{*}{ Años en el servicio } & Menos de 1 año & 14 & 19,2 \\
\hline & De 1 a 2 años & 3 & 4,1 \\
\hline & De 3 a 4 años & 15 & 20,5 \\
\hline & De 5 a 6 años & 15 & 20,5 \\
\hline & Más de 7 años & 26 & 35,6 \\
\hline
\end{tabular}

Tabla 2. Distribución del número de participantes según Calidad del cuidado de enfermería en pacientes con dolor de acuerdo a las tres dimensiones de Donabedian ( $\mathrm{n}=73)$.

\begin{tabular}{lccrrrr}
\hline \multirow{2}{*}{ Calidad del cuidado de enfermería } & \multicolumn{2}{c}{ Estructura } & \multicolumn{2}{c}{ Proceso } & \multicolumn{2}{c}{ Resultado } \\
\cline { 2 - 7 } & Fr & \% & Fr & \% & Fr & \% \\
\hline Calidad máxima (90\% - 100\%) & 54 & 74 & 56 & 76,7 & 57 & 78,1 \\
Calidad regular (80\%-89\%) & 11 & 15 & 7 & 9,6 & 9 & 12,3 \\
Calidad mínima ( $\leq 79 \%)$ & 8 & 11 & 10 & 13,7 & 7 & 9,6 \\
\hline Total & 73 & 100 & 73 & 100 & 73 & 100 \\
\hline
\end{tabular}


Tabla 3. Asociación entre Calidad del cuidado de enfermería en pacientes con dolor y variables sociodemográficas y laborales $(n=73)$.

\begin{tabular}{|c|c|c|c|c|c|c|c|c|}
\hline \multirow{2}{*}{\multicolumn{2}{|c|}{ Variables sociodemográficas y laborales }} & \multicolumn{6}{|c|}{ Calidad del cuidado de enfermería } & \multirow{3}{*}{$\mathbf{p}^{*}$} \\
\hline & & \multicolumn{2}{|c|}{ Calidad máxima } & \multicolumn{2}{|c|}{ Calidad regular } & \multicolumn{2}{|c|}{ Calidad mínima } & \\
\hline & & Fr & $\%$ & Fr & $\%$ & Fr & $\%$ & \\
\hline \multirow{4}{*}{ Edad (años) } & $22-29$ & 9 & 81,8 & 2 & 18,2 & 0 & 0,0 & 0,407 \\
\hline & $30-39$ & 14 & 73,7 & 2 & 10,5 & 3 & 15,8 & \\
\hline & $40-49$ & 13 & 59,1 & 5 & 22,7 & 4 & 18,2 & \\
\hline & 50 y más & 18 & 85,7 & 2 & 9,5 & 1 & 4,8 & \\
\hline \multirow[t]{2}{*}{ Sexo } & Mujer & 44 & 75,9 & 8 & 13,8 & 6 & 10,3 & 0,764 \\
\hline & Hombre & 10 & 66,7 & 3 & 20,0 & 2 & 13,3 & \\
\hline \multirow[t]{2}{*}{ Turno } & Matutino & 30 & 71,4 & 7 & 16,7 & 5 & 11,9 & 0,846 \\
\hline & Vespertino & 24 & 77,4 & 4 & 12,9 & 3 & 9,7 & \\
\hline \multirow[t]{6}{*}{ Servicio } & Cirugía & 6 & 85,7 & 1 & 14,3 & 0 & 0,0 & 0,915 \\
\hline & Medicina interna & 11 & 78,6 & 1 & 7,1 & 2 & 14,3 & \\
\hline & Recuperación & 8 & 66,7 & 2 & 16,7 & 2 & 16,7 & \\
\hline & Urgencias & 16 & 80,0 & 2 & 10,0 & 2 & 10,0 & \\
\hline & Traumatología & 2 & 66,7 & 1 & 33,3 & 0 & 0,0 & \\
\hline & Ginecología & 11 & 64,7 & 4 & 23,5 & 2 & 11,8 & \\
\hline \multirow[t]{6}{*}{ Nivel académico } & Auxiliar de enfermería & 4 & 23,5 & 6 & 35,3 & 7 & 41,2 & 0,000 \\
\hline & Enfermera general & 10 & 90,9 & 1 & 9,1 & 0 & 0,0 & \\
\hline & Especialidad postécnico & 1 & 100,0 & 0 & 0,0 & 0 & 0,0 & \\
\hline & Especialidad de posgrado & 11 & 91,7 & 0 & 0,0 & 1 & 8,3 & \\
\hline & Licenciatura & 27 & 87,1 & 4 & 12,9 & 0 & 0,0 & \\
\hline & Maestría & 1 & 100,0 & 0 & 0,0 & 0 & 0,0 & \\
\hline \multirow[t]{5}{*}{ Antigüedad laboral } & Menos de 3 años & 7 & 87,5 & 1 & 12,5 & 0 & 0,0 & 0,890 \\
\hline & 4-6 años & 8 & 66,7 & 2 & 16,7 & 2 & 16,7 & \\
\hline & 7-9 años & 7 & 58,3 & 3 & 25,0 & 2 & 16,7 & \\
\hline & 10-12 años & 9 & 81,8 & 1 & 9,1 & 1 & 9,1 & \\
\hline & 12 ó más años & 23 & 76,7 & 4 & 13,3 & 3 & 10,0 & \\
\hline \multirow[t]{5}{*}{ Años en el servicio } & Menos de 1 año & 10 & 71,4 & 3 & 21,4 & 1 & 7,1 & 0,702 \\
\hline & De 1 a 2 años & 3 & 100,0 & 0 & 0,0 & 0 & 0,0 & \\
\hline & De 3 a 4 años & 10 & 66,7 & 2 & 13,3 & 3 & 20,0 & \\
\hline & De 5 a 6 años & 10 & 66,7 & 2 & 13,3 & 3 & 20,0 & \\
\hline & Más de 7 años & 21 & 80,8 & 4 & 15,4 & 1 & 3,8 & \\
\hline
\end{tabular}

*Prueba Chi-cuadrada. 
Tabla 4. Análisis de diferencias de las variables sociodemográficas y laborales con la Calidad del cuidado de enfermería en pacientes con dolor $(n=73)$.

\begin{tabular}{|c|c|c|c|}
\hline \multicolumn{2}{|c|}{ Variables sociodemográficas y laborales } & \multirow{2}{*}{$\frac{\mathbf{r}^{*}}{33,32}$} & \multirow{2}{*}{$\frac{\mathbf{p}}{0,027 \ddagger}$} \\
\hline Edad (años) & $22-29$ & & \\
\hline & $30-39$ & 34,05 & \\
\hline & $40-49$ & 30,39 & \\
\hline & 50 y más & 48,52 & \\
\hline \multirow[t]{2}{*}{ Sexo } & Mujer & 37,51 & $0,685 \dagger$ \\
\hline & Hombre & 35,03 & \\
\hline \multirow[t]{2}{*}{ Turno } & Matutino & 37,65 & $0,757 \dagger$ \\
\hline & Vespertino & 36,11 & \\
\hline \multirow[t]{6}{*}{ Servicio } & Cirugía & 45,29 & $0,594 \neq$ \\
\hline & Medicina interna & 39,82 & \\
\hline & Recuperación & 31,96 & \\
\hline & Urgencias & 40,20 & \\
\hline & Traumatología & 29,17 & \\
\hline & Gibecología & 32,44 & \\
\hline \multirow[t]{6}{*}{ Nivel académico } & Auxiliar de enfermería & 17,74 & $0,001 \neq$ \\
\hline & Enfermera general & 44,59 & \\
\hline & Especialidad postécnico & 67,00 & \\
\hline & Especialidad de posgrado & 42,00 & \\
\hline & Licenciatura & 41,42 & \\
\hline & Maestría & 54,00 & \\
\hline \multirow[t]{5}{*}{ Antigüedad laboral } & Menos de 3 ańos & 36,56 & $0,124 \neq$ \\
\hline & 4-6 años & 31,83 & \\
\hline & 7-9 años & 24,79 & \\
\hline & 10-12 años & 42,18 & \\
\hline & 12 ó más años & 42,17 & \\
\hline \multirow[t]{5}{*}{ Años en el servicio } & Menos de 1 año & 36,32 & $0,197 \ddagger$ \\
\hline & De 1 a 2 años & 48,67 & \\
\hline & De 3 a 4 años & 29,97 & \\
\hline & De 5 a 6 años & 31,37 & \\
\hline & Más de 7 años & 43,33 & \\
\hline
\end{tabular}

*rango medio; †Prueba U Mann-Whitney; †Prueba Kruskal-Wallis. 
Tabla 5. Comparación entre pareja de las variables sociodemográficas y laborales de acuerdo a la Calidad del cuidado de enfermería en pacientes con dolor $(n=73)$.

\begin{tabular}{|c|c|c|c|}
\hline Variables & Muestra 1 & Muestra 2 & $\mathbf{p}^{*}$ \\
\hline \multirow[t]{6}{*}{ Edad (años) } & $40-49$ & $22-29$ & 1,000 \\
\hline & $40-49$ & $30-39$ & 1,000 \\
\hline & $40-49$ & 50 y más & 0,029 \\
\hline & $22-29$ & $30-39$ & 1,000 \\
\hline & $22-29$ & 50 y más & 0,314 \\
\hline & $30-39$ & 50 y más & 0,180 \\
\hline \multirow[t]{15}{*}{ Nivel académico } & Auxiliar de enfermería & Licenciatura & 0,003 \\
\hline & Auxiliar de enfermería & Enfermera general & 0,015 \\
\hline & Auxiliar de enfermería & Especialidad de posgrado & 0,034 \\
\hline & Auxiliar de enfermería & Especialidad postécnico & 0,345 \\
\hline & Auxiliar de enfermería & Maestría & 1,000 \\
\hline & Licenciatura & Enfermera general & 1,000 \\
\hline & Licenciatura & Especialidad de posgrado & 1,000 \\
\hline & Licenciatura & Especialidad postécnico & 1,000 \\
\hline & Licenciatura & Maestría & 1,000 \\
\hline & Especialidad de posgrado & Enfermera general & 1,000 \\
\hline & Especialidad de posgrado & Especialidad postécnico & 1,000 \\
\hline & Especialidad de posgrado & Maestría & 1,000 \\
\hline & Especialidad postécnico & Enfermera general & 1,000 \\
\hline & Maestría & Enfermera general & 1,000 \\
\hline & Maestría & Especialidad postécnico & 1,000 \\
\hline
\end{tabular}

*Prueba Kruskal-Wallis a posteriori, cuando se rechaza la hipótesis nula.

\section{DISCUSIÓN}

La forma de medir la calidad del cuidado desde el punto de vista de Donabedian dependerá del sistema de salud que tenga cada país, sin embargo, las dimensiones de la calidad desde el enfoque de estructura-proceso-resultado deben dirigir los indicadores nacionales, principalmente para enfermería. Como profesionales dedicados a la gestión del cuidado, esto permitiría estructurar acciones que tengan un impacto positivo en la salud de los usuarios con dolor, porque definir estándares de productos, servicios y procesos del trabajo enfermero, se ve reflejado en la calidad del cuidado de enfermería que brindan las instituciones de salud ${ }^{(24-26)}$.
En este estudio, la calidad del cuidado de enfermería alcanzó una calidad máxima, principalmente en el resultado más que en la estructura y el proceso, tal como Voyce et al. ${ }^{(27)}$, quizás porque la responsabilidad clínica de enfermería va más allá de los procesos de administración hospitalaria, está centrada, principalmente, en y para el cuidado del paciente, que respeta y valora sus preferencias y necesidades. Esto supone que los procesos de calidad en los hospitales deben incluir indicadores sensibles a la actividad de enfermería, que ayuden a replantear lo que falta y en lo que se debe profundizar de tal forma que se logre recuperar la esencia de enfermería: el cuidado ${ }^{(28)}$.

En este estudio el personal de enfermería del sexo femenino, de mayor edad y el turno vespertino 
presentó una calidad máxima. Esto también lo han reportado otros autores, donde el sexo y el turno juegan un papel relevante en la atención de enfermería, debido a que una filosofía de servicio $y$ el trabajo en equipo permiten satisfacer las demandas del paciente y con ello, mantener la calidad en la atención en cada uno de los servicios del hospital ${ }^{(29)}$.

Como lo manifiestan también otros estudios ${ }^{(30-32)}$, el nivel académico fue una de las variables con la que se asoció la calidad del cuidado de enfermería en pacientes con dolor. Esto indica que se requiere personal preparado para la atención de calidad de este tipo de pacientes, y esa preparación impactaría en la estructura, el proceso y, por supuesto, el resultado de la atención de las instituciones de salud.

Vinculado a estos resultados está lo planteado por Benner, en su Modelo de Dreyfus de adquisición de habilidades, por cierto, aplicable al ámbito clínico, donde el personal de enfermería requiere de un desarrollo profesional continuo, que le permita perfeccionar sus competencias y avanzar desde una atapa de principiante hasta llegar a la etapa de experto/a. Las instituciones de salud deben mantener capacitaciones y con ello aumentar los niveles de estudio del personal de enfermería ${ }^{(33)}$.

Se observa además que la calidad del cuidado de enfermería depende de la edad. Esta situación también puede ser explicada por la teoría de Banner en la etapa de experto, porque describe que conforme el personal de enfermería desarrolla su experiencia, adquiere habilidades y conocimiento, hace del cuidado una práctica segura y rápida en beneficio de sus pacientes con dolor ${ }^{(33)}$.

Es importante reconocer que a cierta edad, los/ as enfermeros/as poseen aspectos que les permiten captar la situación de forma intuitiva en función de los conocimientos incorporados, cuentan con habilidades necesarias y mayor confianza en sí mismos, están satisfechos con sus logros y consideran que las victorias son el punto de partida para un nuevo objetivo que exige elevar su desempeño y prepararse para situaciones clínicas más complejas. Asimismo, los/as enfermeros/as mayores con experiencia tienen conocimientos que no están escritos en los libros de texto, sino que los han adquirido en las situaciones que enfrentan y cuidan con más dedicación al paciente ${ }^{(34)}$. El personal de enfermería es pieza clave en el proceso de gestión, sus intervenciones están dirigidas a lograr un cuidado de calidad máxima, con el apoyo de políticas relacionadas a la atención de salud, tal como sucede en Brasil, donde la calidad del cuidado de enfermería en el control de infecciones, desde la perspectiva teórica de Donabedian, favorece la asistencia sanitaria y una cultura de asistencia segura en los servicios de salud ${ }^{(35)}$.

El manejo de una atención para la calidad del cuidado es relevante, considerando que la calidad de la atención de salud tiende a impactar tanto en la práctica del profesional y en la calidad de vida del paciente, como en la unidad de salud donde se presta el servicio ${ }^{(36)}$. Atendiendo a Donabedian, el secreto de la calidad, en este caso la calidad del cuidado de enfermería del paciente con dolor, es el amor a los pacientes, el amor a la profesión y el amor a $\operatorname{Dios}^{(37)}$.

Una de las limitaciones de este estudio fue el muestreo por conveniencia, que limita hacer generalizaciones, sin embargo, a través del cálculo de la muestra poblaciones finitas para obtener el mínimo de enfermeros que participarían en este estudio y la verificación del llenado de forma completa de los instrumentos hasta completar la muestra minimizó el sesgo de la selección. Otra limitación fue el acceso al personal de enfermería debido a la carga de trabajo a la que se ve sometido y que en su momento dificultó responder el instrumento. Otra limitante fue la falta de estudios previos con esta temática, pero este estudio es una oportunidad para abrir nuevas líneas de conocimiento en la calidad del cuidado de enfermería.

\section{CONCLUSIÓN}

La calidad del cuidado de enfermería en pacientes con dolor depende del nivel académico y la edad, lo que requiere una especial atención de todos los involucrados, debido a que es un proceso dinámico que difícilmente puede abordarse de la misma forma en todas las áreas hospitalarias y turnos que la componen, debido a los diferentes factores que en su momento favorecen o dificultan dicho proceso. Es necesario plantear entonces estrategias a través de la educación y desarrollo profesional continuo, que mejoren los resultados de la calidad del cuidado de enfermería en estos pacientes y 
desde luego la calidad de vida del usuario. Además, los profesionales de salud deben adaptarse a un nuevo escenario, que conlleva grandes desventajas y nuevos riesgos. Estos profesionales serán de gran importancia en aquellos pacientes frágiles, que requieren una atención especializada en entornos

\section{REFERENCIAS}

1. Syed S, Leatherman S, Mensah N, Neilson M, Kelley E. Improving the quality of health care across the health system. Bull World Health Organ [Internet]. 2018 [citado 17 ago 2019]; 96: 799. Disponible en: http://dx.doi.org/10.2471/ BLT.18.226266

2. Ayuso D, De Andrés B. Gestión de la calidad de cuidados de enfermería. Madrid: Ediciones Díaz de Santos; 2015. 432 p.

3. Donabedian A. The definition of quality and approaches to its assessment. Ann Arbor, Michigan: Regents of the University of Michigan; 1980. $176 \mathrm{p}$.

4. Harvey C, Thompson S, Pearson M, Willis E, Toffoli L. Missed nursing care as an 'art form': The contradictions of nurses as carers. Nurs Inq [Internet]. 2017 Jul [citado 17 ago 2019]; 24(3). Disponible en: https://doi.org/10.1111/nin.12180

5. Naranjo Y, Concepción JA, Rodríguez M. La teoría Déficit de autocuidado: Dorothea Elizabeth Orem. Gaceta Médica Espirituana [Internet]. 2017 [citado 5 oct 2020]; 19(3): 1-11. Disponible en: https://www.medigraphic.com/cgi-bin/new/ resumen.cgi?IDARTICULO $=77397$

6. Luengo C, Sanhueza O. Condiciones de trabajo y su relación con la calidad del cuidado y salud del profesional de enfermería. Med segur trab [Internet]. 2016 [citado 17 ago 2018]; 62(245): 368-80. Disponible en: http://scielo.isciii.es/pdf/ mesetra/v62n245/08_revision.pdf

7. Koy V, Yunibhand J, Angsuroch Y, Fisher ML. Relationship between nursing care quality, nurse staffing, nurse job satisfaction, nurse practice environment, and burnout: literature review. Int J Res Med Sci [Internet]. 2015 [citado 17 ago 2018]; 3(8): 1825-31. Disponible en: http://dx.doi. org/10.18203/2320-6012.ijrms20150288

8. Owens LD, Koch RW. Understanding quality patient care and the role of the practicing nurse. Nurs Clin North Am [Internet]. 2015 [citado 17 ago 2018]; 50(1): 33-43. Disponible en: 10.1016/j. de mejora continua, en donde se van estableciendo metas y objetivos que, una vez logradas, conducen a buscar nuevas mejoras de calidad del cuidado de enfermería en la estructura, proceso y resultado de las instituciones de salud.

cnur.2014.10.003

9. Zarzycka D, Bartoń E, Mazur A, Turowski K. Socio-demographic and medical factors associated with patients' satisfaction with nursing care and their perception of pain. Ann Agric Environ Med [Internet]. 2019 [citado 17 ago 2019]; 26(2): 298303. Disponible en: https://doi.org/10.26444/ aaem/90385

10. Torres CCC, Páez EAN, Rincón SL, Rosas BD, Mendoza MEP. Reproducibilidad del cuestionario: calidad de cuidados de enfermería en pacientes hospitalizados. Rev Cuid [Internet]. 2016 [citado 19 jul 2019]; 7(2): 1338-44. Disponible en: https:// doi.org/10.15649/cuidarte.v7i2.339

11. Alonso R, Blanco Ramos MA, Ganoso P. Validación de un cuestionario de calidad de cuidados de enfermería. Rev Calid Asist [Internet]. 2005 [citado 17 ago 2018]; 20(5): 24650. Disponible en: https://doi.org/10.1016/S1134282X(05)75092-2

12. Thomas LH, McColl E, Priest J, Bond S, Boys R. Newcastle satisfaction with nursing scales: an instrument for quality assessments of nurse care. Qual health care [Internet]. 1996 [citado 17 ago 2018]; 5 (2): 62-72. Disponible en: https://doi. org/10.1136/qshc.5.2.67

13. Salas SBA, Galiano GMA. Percepción de enfermeras y familiares de pacientes sobre conductas de cuidado importantes. Cienc enferm [Internet]. 2017 [citado 17 ago 2018]; 23(1): 35-44. Disponible en: http://dx.doi.org/10.4067/S071795532017000100035

14. Larson P. Important nurse caring behaviors perceived by patients with cancer. Oncol Nurs Forum [Internet]. 1984 [citado 17 ago 2018]; 11(6): 46-50. Disponible en: $10.1891 / 9780826171122.0018$

15. Orrego SS, Ortiz ZAC. Calidad del cuidado de enfermería. Invest Educ Enferm [Internet]. 2001 [citado 17 ago 2018]; 19(2): 78-83. Disponible en: https://revistas.udea.edu.co/index.php/iee/article/ view/16813/14565

16. González KG, Zambrano CM, Fleitas GD. Ética 
y bioética en los profesionales de enfermería. Dominio de las ciencias [Internet]. 2016 [citado 17 ago 2018]; 2: 106-19. Disponible en: https://dialnet. unirioja.es/servlet/articulo?codigo $=6325816$

17. Ruiz de ChMH. Reflexiones sobre el binomio enfermería y bioética. Gaceta Conbioética [Internet]. 2016 [citado 17 ago 2018]; 6(22): 3-11. Disponible en: http://www.conbioeticamexico.salud.gob.mx/interior/gaceta_conbioetica/ Gaceta_22/Gaceta_22_Enfermeria.pdf

18. Donabedian A. Quality of care: problems of measurement II. Some issues in evaluating the quality of nursing care. Am J Public Health Nations Health [Internet]. 1969 [citado 17 ago 2018]; 59(10): 1833-36. Disponible en: 10.2105/ ajph.59.10.1833

19. García HML, Arana GB, Salvador GV, Salvador MC, Gutiérrez LA, Hernández OY. Propuesta de validación de indicadores y estándares para el manejo del dolor en el paciente. Esc Anna Nery [Internet]. 2009 [citado 17 ago 2018]; 13 (1): 8490. Disponible en: https://doi.org/10.1590/S141481452009000100012

20. International Association for the Study of Pain. IASP Terminology [Internet]. (C) 2018 [citado 9 abr 2020]. Disponible en: https://www.iasp-pain. org/terminology?navItemNumber=576\#Pain

21. García HML, Hernández OY, Arana GB, García OA. Calidad del cuidado de enfermería en pacientes hospitalizados con dolor. Revista Horizontes en Salud [Internet]. 2011 [citado 17 ago 2018]; 3: 55-69. Disponible en: http://web.uaemex.mx/ revistahorizontes/docs/revistas/Vol3/CALIDAD_ DEL_CUIDADO_DE_ENFERMERIA.pdf

22. World Medical Association, Inc. WMA Declaration of Helsinki - Ethical Principles for Medical Research Involving Human Subjects [Internet]. 2018 Jul 9 [citado 17 ago 2018]. Disponible en: https://www.wma.net/policiespost/wma-declaration-of-helsinki-ethicalprinciples-for-medical-research-involving-humansubjects/

23. México. Reglamento de la Ley General de Salud en materia de investigación para la salud [Internet]. Última Reforma DOF 02-04-2014, Nuevo Reglamento publicado en el Diario Oficial de la Federación el 6 de enero de 1987. [citado 20 abr 2020]. Disponible en: http://www.diputados.gob. $\mathrm{mx} /$ LeyesBiblio/regley/Reg_LGS_MIS.pdf

24. Beaussier AL, Demeritt D, Rothstein $H$, Griffiths A. Steering by their own lights: Why regulators across Europe use different indicators to measure healthcare quality. Health Policy [Internet]. 2020 [citado 17 may 2020]; 124(5): 501-10. Disponible en: https://doi.org/10.1016/j. healthpol.2020.02.012

25. Zaslansky R, Chapman R, Meissner W. ¿Qué se puede hacer para mejorar el manejo del dolor perioperatorio? Rev Mex Anest [Internet]. 2019 [citado 17 may 2020]; 42(3): 149-52. Disponible en: https://www.medigraphic.com/cgi-bin/new/ resumen.cgi?IDARTICULO $=88607$

26. Febré N, Mondaca K, Méndez P, Badilla V, Soto P, Ivanovic $\mathrm{P}$ et al. Calidad de enfermería: su gestión, implementación y medición. Rev Med Clin Condes [Internet]. 2018 [citado 5 oct 2020]; 29(3): 278-287. Disponible en: https://doi.org/10.1016/j. rmclc.2018.04.008

27. Voyce J, Gouveia MJ, Medinas MA, Santos AS, Ferreira RF. A Donabedian Model of the Quality of Nursing Care from Nurses' Perspectives in a Portuguese Hospital: A Pilot Study. J Nurs Meas [Internet]. 2015 [citado 17 ago 2018]; 23(3): 474484. Disponible en: https://doi.org/10.1891/10613749.23.3.474

28. Somoza HB, Cano GMV, Guerra LP. Farmacología en Enfermería: Casos clínicos. Madrid: Editorial Médica Panamericana, 2012. 21 p.

29. Haftu M, Girmay A, Gebremeskel M, Aregawi G, Gebregziabher D, Robles C. Commonly missed nursing cares in the obstetrics and gynecologic wards of Tigray general hospitals; Northern Ethiopia. PLoS ONE [Internet]. 2019 [citado 17 abr 2020]; 14(12): e0225814. Disponible en: https://doi.org/10.1371/journal.pone.0225814

30. Cho SH, Lee JY, You SJ, Song KJ, Hong KJ. Nurse staffing, nurse's prioritization, missed care, quality of nursing care, and nurse outcomes. Int J Nurs Pract [Internet]. 2020 [citado 17 may 2020]; 26(1): e12803. Disponible en: https://doi.org/10.1111/ ijn.12803

31. Flores-Alpízar CA, Ponce-Moreno R, Ruíz-Jalomo MR, Corral-Quiroz RJ. Factores relacionados con la calidad de atención de enfermería. Rev Enferm Inst Mex Seguro Soc [Internet]. 2015 [citado 17 ago 2018]; 23(3): 143-8. Disponible en: http:// revistaenfermeria.imss.gob.mx/editorial/index. php/revista_enfermeria/rt/printerFriendly/56/101

32. Liu X, Zheng J, Liu K, Gedney Baggs J, Liu J, Wu $\mathrm{Y}$ et al. Hospital nursing organizational factors, nursing care left undone, and nurse burnout as predictors of patient safety: A structural equation modeling analysis. Int J Nurs Stud [Internet]. 2018 [citado 17 may 2020]; 86: 82-9. Disponible en: https://doi.org/10.1016/j.ijnurstu.2018.05.005

33. Benner P. From Novice to Expert. Am J Nurs [Internet]. 1982 [citado 9 oct 2020]; 82(3): 4027. Disponible en: http://www.medicalcenter. virginia.edu/internet/therapy-services/3\%20-\%20 Benner\%20-\%20Novice\%20to\%20Expert-1.pdf 
34. Rodríguez S, Cárdenas M, Pacheco AL, Ramírez M, Ferro N, Alvarado E. Reflexión teórica sobre el arte del cuidado. Enfermería Universitaria [Internet]. 2017 [citado 9 oct 2020]; 14(3): 191-8. Disponible en: https://doi.org/10.1016/j.reu.2017.05.004

35. Nascimento T, Lopes F, Silvino Z, Ferreira D, Políticas de controle de infecção no Brasil e qualidade da assistência de enfermagem: reflexóes necessárias. Research, Society and Development [Internet]. 2020 [citado 17 may 2020]; 9(5): e56953178. Disponible en: http://dx.doi.org/10.33448/rsd- v9i5.3178

36. Lopes F, Silvino Z, Dutra F, Balbino C, De Souza C, Dos Santos L. Care management for patients with venous ulcers. J Nurs UFPE [Internet]. 2019 [citado 17 may 2020]; 13: e243017. Disponible en: http://dx.doi.org/10.5205/1981-8963.2019.243017

37. Backer LA. Rediscovering the Secret of Quality. Fam Pract Manag [Internet]. 2019 [citado 17 may 2020]; 26(2): 4. Disponible en: https://www.aafp. org/fpm/2019/0300/p4.html 